\title{
Modelos teóricos utilizados por enfermeiros para avaliação da família: reflexão teórica
}

\author{
Theoretical models used by nurses for family assessment: theoretical reflection
}

Modelos teóricos utilizados por enfermeros para la evaluación familiar: reflexión teórica

Thais Vilela de Sousa ${ }^{1 *}$, Cecília Sousa Macêdo ${ }^{1}$, Ariana Fidelis Alves Santana ${ }^{2}$, Maria Luiza Rêgo Bezerra $^{3}$, Francidalma Soares Sousa Carvalho Filha ${ }^{4}$, Mayara Cândida Pereira ${ }^{3}$, Ricardo Costa da Silva ${ }^{1}$, Meillyne Alves dos Reis ${ }^{1}$, Micaelle Costa Gondim¹, Iel Marciano de Moraes Filho ${ }^{3}$.

\section{RESUMO}

Objetivo: identificar a produção científica no período de 10 anos completos, a partir da implementação da Atenção Básica no Brasil, sobre os modelos teóricos utilizados e que influenciaram os enfermeiros na avaliação de famílias no brasil e no mundo. Métodos: Trata-se de um estudo teórico-reflexivo, construído com base na leitura crítica da avaliação dos modelos familiares pelos profissionais de enfermagem, em estudos científicos, que referenciam as avaliações familiares como fatores preditores na melhora significativa no cuidado direto ao paciente. Essa construção teórica aproxima-se da abordagem qualitativa, tendo em vista a interpretação e análise dos elementos teóricos obtidos por meio de uma revisão integrativa. Resultados: Foram utilizados 20 artigos e encontrados 19 modelos. Os Modelos Calgary, o APGAR de família e o Genograma e Ecomapa são mais comumente utilizados na prática clínica do enfermeiro. Considerações Finais: A utilização de modelos teóricos de avaliação propicia melhoria na qualidade do cuidado dispensado bem como a sistematização da assistência, a integração da família e suas relações no cuidar promovendo um fortalecimento familiar.

Palavras-chave: Enfermagem familiar, Relações familiares, Família, Modelos teóricos, Modelos de enfermagem.

\begin{abstract}
Objective: To identify the scientific production in the period of 10 complete years, from the implementation of Primary Care in Brazil, on the theoretical models used and that influenced nurses in the evaluation of families in Brazil and worldwide. Methods: This is a theoretical-reflexive study, built on the critical reading of the evaluation of family models by nursing professionals, in scientific studies, which refer to family evaluations as predictive factors for significant improvement in direct patient care. This theoretical construction approaches the qualitative approach, in view of the interpretation and analysis of the theoretical elements obtained through an integrative review. Results: We used 20 articles and found 19 models. Calgary Models, family APGAR, and Genogram and Ecomapa are most commonly used in clinical nursing practice. Conclusion: The use of theoretical evaluation models provides improvements in the quality of care provided as well as the systematization of care, the integration of the family and their relationships in care, promoting family strengthening.
\end{abstract}

Keywords: Family nursing, Family relationships, Family, Theoretical Models, Nursing models.

\footnotetext{
1 Universidade Federal de Goiás (UFG), Goiânia-GO. * E-mail: ielfilho@yahoo.com

2 Pontifícia Universidade Católica de Goiás (PUC-GO), Goiânia-GO.

3 Universidade Paulista Campus Brasília (UNIP), Brasília-DF.

${ }^{4}$ Universidade Estadual Do Maranhão (UEMA), Balsas-MA.
} 


\section{RESUMEN}

Objetivo: identificar la producción científica en el período de 10 años completos, desde la implementación de la Atención Primaria en Brasil, sobre los modelos teóricos utilizados y que influyeron en los enfermeros en la evaluación de las familias en Brasil y en todo el mundo. Métodos: Este es un estudio teórico-reflexivo, construido sobre la lectura crítica de la evaluación de modelos familiares por profesionales de enfermería, en los estudios científicos, que se refieren a las evaluaciones familiares como factores predictivos para una mejora significativa en la atención directa al paciente. Esta construcción teórica se acerca al enfoque cualitativo, en vista de la interpretación y el análisis de los elementos teóricos obtenidos a través de una revisión integradora. Resultados: Utilizamos 20 artículos y encontramos 19 modelos. Los modelos de Calgary, APGAR familiar y Genograma y Ecomapa son los más utilizados en la práctica clínica de enfermería. Conclusión: El uso de modelos de evaluación teórica proporciona una mejora en la calidad de la atención brindada, así como en la sistematización de la atención, la integración de la familia y sus relaciones en la atención, promoviendo el fortalecimiento familiar.

Palabras clave: Enfermería familiar, Relaciones familiares, Familia, Modelos teóricos, Modelos de enfermería.

\section{INTRODUÇÃO}

A família pode ser compreendida como um agrupamento humano formado por duas ou mais pessoas com ancestrais em comum ou ligações afetivas que, geralmente, vivem numa mesma casa. Consoante com Lopes MSL e Marcon SS (2009), a mesma vem fortalecendo-se como instituição social tanto pelo seu papel de receber e acolher o indivíduo quanto pelo seu caráter nucleador, e não só no âmbito dos movimentos sociais, como também no que diz respeito às políticas públicas de saúde.

Assim, a família é um sistema de saúde para seus integrantes, pois nela encontra - se proteção em que seus membros se sentem unidos por laços de amor e afeto, que ao se verem fragilizados por uma situação de doença, buscam dentro desta unidade familiar, seus conhecimentos culturais, crenças e práticas com intuito da recuperação do bem-estar (LOPES MSL e MARCON SS, 2009).

Além do mais, as famílias se constituem por membros que são considerados pelos próprios entes familiares, sendo vista como um sistema familiar e dentro deste, existem subsistemas compostos pela relação pais-filhos, cônjuges e irmãos; e ainda está relacionada a subsistemas mais amplos como a vizinhança, as organizações sociais e as comunidades religiosas (WRIGHT LM e LEAHEY M, 2012).

Em se tratando do processo saúde e doença, geralmente os membros familiares compartilham os cuidados, a internação e o tratamento do ente querido, passando a ser o grande elo entre paciente e profissionais de saúde, fornecendo informações importantes, suporte social e compartilhando o processo de cuidar.

Assim, é essencial que a equipe de enfermagem se aproxime da família, estabelecendo vínculos e ofertando a atenção necessária à melhoria das condições de vida e saúde (LOPES MSL e MARCON SS, 2009; WRIGHT LM e LEAHEY M, 2012; MORAES IM FILHO, et al., 2019).

Desta maneira, quando algum membro da família apresenta problemas de saúde, a família precisa se reorganizar e neste contexto, os enfermeiros devem direcionar a assistência para a orientação e os cuidados necessários. Neste ínterim, tais profissionais podem utilizar a avaliação dos modelos familiares, impulsionados após a implementação do Programa Saúde da Família (PSF) pelo o Ministério da Saúde em 1994.

O programa tem o objetivo de reorganizar a prática assistencial, substituindo o modelo médicohegemônico, por um modelo baseado na promoção da qualidade de vida e intervenção nos fatores que colocam em risco a saúde da comunidade, por meio de ações programáticas e intersetoriais, com a participação da comunidade e através da coordenação da equipe de enfermagem, tanto de forma gerencial quanto na implementação das políticas nacionais de saúde, a mesma na atualidade denominada Estratégia 
Saúde da Família (ESF) (SEOANE AF e FORTES PAC, 2009; FIGUEIREDO MHJS e MARTINS MMFS, 2010; WRIGHT LM e LEAHEY M, 2012;FILHOIM DE M, et al., 2018).

Destaca-se que a partir da implementação da consulta de enfermagem, realiza-se a avaliação da família com vistas a identificar, através de suas relações, as situações de saúde/doença, prescrevendo e desenvolvendo ações de enfermagem de apoio, promoção, prevenção, recuperação e reabilitação da saúde (CHRISTOFFEL MM, et al., 2008; FIGUEIREDO MHJS e MARTINS MMFS, 2010; CICOLINI G. et al., 2012; FILHO IM DE M, et al., 2019).

Ouvir a família na entrevista permite a exposição dos problemas e proporciona subsídio para orientar durante a avaliação, ajudando significativamente a detecção e solução de problemas (FILIZOLA CLA, et al., 2003).

Desta forma, as intervenções de enfermagem devem promover a autonomia do sistema familiar, baseando-se na parceria com a família e visando fortalecer suas competências e independências (FIGUEIREDO MHJS e MARTINS MMFS, 2010).

Para facilitar tanto o processo de aprendizagem e de cuidado, quanto a prática assistencial propriamente dita, existem modelos teóricos que auxiliam na avaliação das famílias e que também podem se enquadrar nas diversas situações do dia-a-dia do enfermeiro, ampliando seu universo de sua atuação (WRIGHT LM e LEAHEY M, 2012).

A avaliação da família por enfermeiros é uma atribuição essencial, concentrando-se na relação de reciprocidade entre família e profissionais, com o intuito de esclarecer o impacto da doença sobre a dinâmica familiar e a influência da interação familiar sobre o curso da doença (CHRISTOFFEL MM, et al., 2008; FIGUEIREDO MHJS e MARTINS MMFS, 2010; WRIGHT LM e LEAHEY M, 2012; CICOLINI G, et al., 2012; FILHO IM DE M, et al., 2019).

Portanto, é essencial que o enfermeiro e os demais profissionais adquiram conhecimento em relação ao universo familiar, atendendo adequadamente às necessidades do outro (SEOANE AF e FORTES PAC, 2009); requerendo a utilização de modelos avaliativos que permitam a realização de cuidados orientados tanto para a coleta de dados como para o planejamento e implantação das intervenções (FIGUEIREDO MHJS e MARTINS MMFS, 2010).

Destaca-se que o objetivo do estudo foi identificar a produção científica no período de 10 anos completos, a partir da implementação da Atenção Básica no Brasil, sobre os modelos teóricos utilizados e que influenciaram os enfermeiros na avaliação de famílias no brasil e no mundo.

Esta investigação se justifica pela escassez de estudos relativos à padronização de instrumentos de avaliação de famílias por enfermeiros.

Além disso, quando a enfermagem elabora teorias, identificam padrões, os nomeia, cria seus métodos de avaliação e intervenção, se fortalece com ciência, ganha respeito e representatividade perante outras áreas do saber, instiga o desenvolvimento de uma assistência mais humana, individualizada, integral e equânime, quer direcionada à pessoa, família ou comunidade.

\section{MÉTODOS}

Trata-se de um estudo teórico-reflexivo, construído com base na leitura crítica da avaliação dos modelos familiares pelos profissionais de enfermagem, em estudos científicos, que referenciam as avaliações familiares como fatores preditores na melhora significativa no cuidado direto ao paciente.

Essa construção teórica aproxima-se da abordagem qualitativa, tendo em vista a interpretação e análise dos elementos teóricos obtidos por meio de uma revisão integrativa da literatura, onde foram seguidas as etapas de: identificação do tema, seleção da hipótese ou questão de pesquisa, estabelecimento de critérios de inclusão e exclusão, definição das informações a serem extraídas, avaliação dos estudos incluídos na revisão, interpretação dos resultados, apresentação da revisão com síntese do conhecimento (MENDES KDS, et al., 2008). 
O percurso metodológico incluiu primeiramente levantamento nas bases de dados da literatura científica: Medical Literature Analysis and Retrieval System Online (MEDLINE) via National Library of Medicine (NLM/Pubmed), Scientific Electronic Library Online (Scielo) e Biblioteca Virtual em Saúde (BVS).

Foram critérios de inclusão: estudos publicados em línguas portuguesa, inglesa e espanhola, disponíveis na íntegra on-line. Excluíram-se as teses, dissertações e editoriais. Para a busca dos estudos aos quais compuseram esta revisão, empregou-se os descritores indexados em inglês e português, que foram consultados nos Descritores em Ciências da Saúde - DeCS e no Medical Subject Headings - MESH (Quadro 1).

Quadro 1 - Estratégias de busca utilizadas nas bases de dados BVS, PUBMED e SCIELO. Goiânia, Brasil, 2019.

\begin{tabular}{|c|c|c|c|c|c|}
\hline $\begin{array}{l}\text { Base de } \\
\text { dados }\end{array}$ & Estratégia de busca & Resultados & Filtrados & Selecionados & $\begin{array}{l}\text { Repetição } \\
\text { em bases }\end{array}$ \\
\hline $\begin{array}{l}\text { BVS } \\
\text { (Decs) }\end{array}$ & 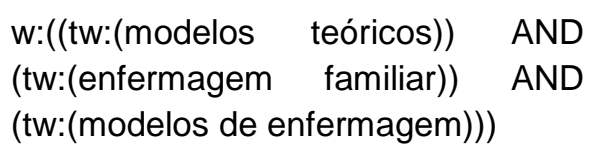 & 222 & 66 & 2 & 4 \\
\hline $\begin{array}{l}\text { BVS } \\
\text { (Decs) }\end{array}$ & $\begin{array}{l}\text { (tw:(theoretical models)) AND (tw:( } \\
\text { family nursing)) AND (tw:(nursing } \\
\text { models)) }\end{array}$ & 861 & 262 & 3 & 4 \\
\hline $\begin{array}{l}\text { PubMed } \\
\text { (MeSH) }\end{array}$ & 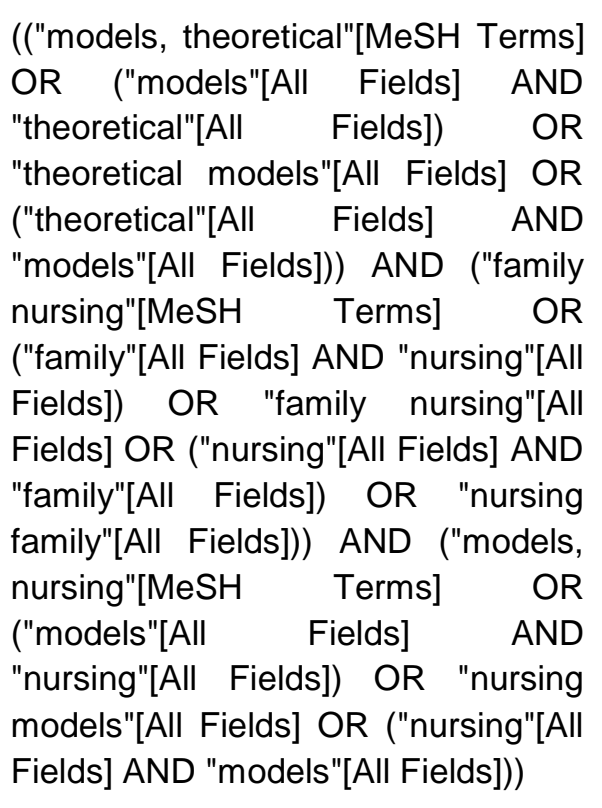 & 3757 & 2392 & 9 & - \\
\hline $\begin{array}{l}\text { Scielo } \\
\text { (Decs) }\end{array}$ & 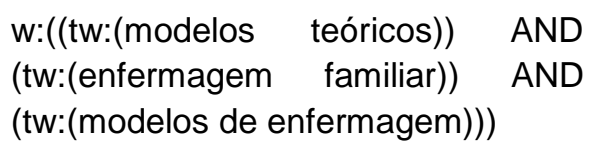 & 2 & 2 & 2 & 4 \\
\hline $\begin{array}{l}\text { Scielo } \\
\text { (Decs) }\end{array}$ & $\begin{array}{l}\text { (theoretical models ) AND (nursing } \\
\text { family ) AND (nursing models) }\end{array}$ & 10 & 10 & 8 & 4 \\
\hline Total & & 4852 & 2722 & 24 & 20 \\
\hline
\end{tabular}

Fonte: Sousa TV, et al., 2019. 
Foram selecionados cinco descritores com base nos Descritores em Ciências da Saúde (DECS): "enfermagem familiar", "relações familiares", "família", "modelos teóricos" e "modelos de enfermagem", os quais foram combinados de três a três, com a finalidade de encontrar o maior número de resultados adequados para o estudo. Sendo assim, a partir das combinações dos descritores. Os que mais se adequaram foram "modelos teóricos/theoretical models", "enfermagem familiar/nursing family" e "modelos de enfermagem/nursing models" pelo operador booleano "and". Também foi aplicado recorte temporal de 1994 a 2015 em consideração ao período de implementação da atenção básica em saúde no Brasil, desta forma houve estímulo para a busca e conhecimento dos modelos teóricos de avaliação familiar por enfermeiros no Brasil e no Mundo.

Estas obras foram ainda analisadas em três etapas: primeiramente por título e resumo sendo avaliados 100 artigos; os mesmos procederam para análise de conteúdo, no intuito de selecionar somente artigos que realmente contemplavam algum modelo teórico de avaliação restando 24 artigos ainda sendo excluídos quatro por repetição nas bases de dados Scielo e BVS. Após esse processo, os 20 artigos restantes sofreram avaliação de conteúdo com interpretação, segregação e síntese dos modelos teóricos utilizados.

\section{RESULTADOS E DISCUSSÃO}

Pelo método de análise desta revisão integrativa, a segregação identificou os seguintes modelos de avalição familiar: sustentados na Teoria de Adaptação de Roy, Saúde da Família (FSM), Parental, APGAR de Família, modelo Calgary, Modelo de análise de depressão em cuidadores, modelo de resiliência, Family Background Questionnarie (FBQ), Critical Care Family Inventory (CCFNI), Genograma e Ecomapa, Entrevista Familiar Estruturada (EFE), Family Assessment Device (FAD), Family Adaptability and Cohesion Evaluation Scales (FACES III), Family Environment Scale (FES), Family Assessment Measure (FAM), Modelo do Cuidado Centrado na Famílma, Questionário de Dinâmica Familiar e Modelo de Neuman como descrito pelo Quadro 2. 


\section{Revista Eletrônica Acervo Saúde / Electronic Journal Collection Health ｜ ISSN 2178-2091}

Quadro 2 - Quadro sinóptico dos modelos de avaliação de família identificados na literatura em publicações do período de 1994 a 2015. Brasília-DF, 2019.

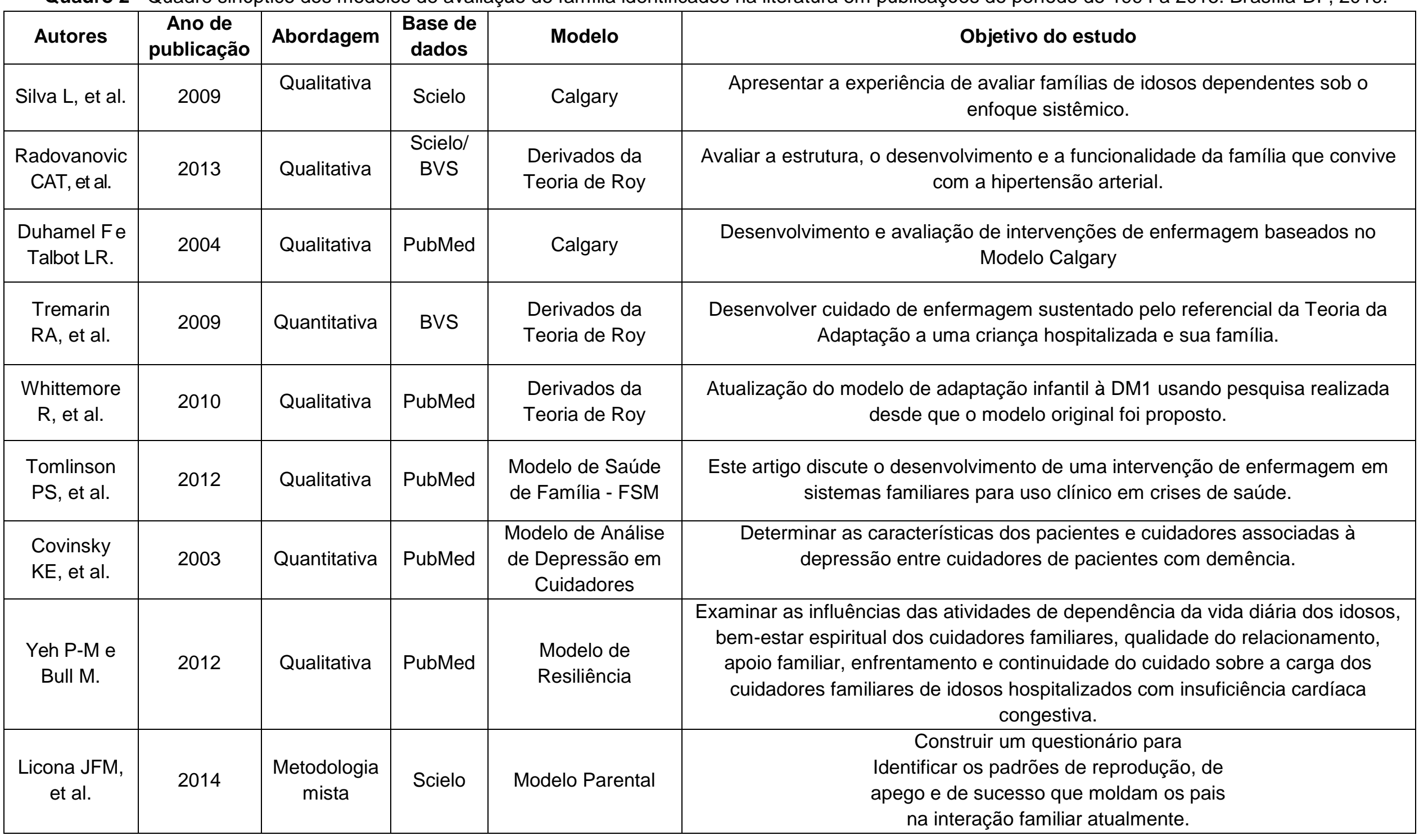

REAS/EJCH | Vol.12(4) | e2614 | DOI: https://doi.org/10.25248/reas.e2614.2020 Página 6 de 15 
Revista Eletrônica Acervo Saúde / Electronic Journal Collection Health ISSN 2178-2091

\begin{tabular}{|c|c|c|c|c|c|}
\hline $\begin{array}{l}\text { Monteiro IS } \\
\text { e Maia AC. }\end{array}$ & 2010 & Qualitativa & $\begin{array}{l}\text { Scielo } \\
\text { /BVS }\end{array}$ & $\begin{array}{l}\text { Family Background } \\
\text { Questionnarie - } \\
\text { FBQ/ Adaptado }\end{array}$ & Adaptação para o português brasil Family Background Questionnari \\
\hline $\begin{array}{l}\text { Maetínez } \\
\text { SG, et al. }\end{array}$ & 2001 & Qualitativa & Scielo & $\begin{array}{l}\text { Critical Care Family } \\
\text { Needs Inventory- } \\
\text { CCFNI }\end{array}$ & $\begin{array}{l}\text { O objetivo do presente estúdio } \\
\text { é adaptar e validar a versão breve do Questionário de } \\
\text { Necessidades dos Familiares de Pacientes de Cuidados } \\
\text { Intensivos (CCFNI) em uma música em espanhol. }\end{array}$ \\
\hline $\begin{array}{c}\text { Silva MJ, et } \\
\text { al. }\end{array}$ & 2014 & Quantitativa & $\begin{array}{l}\text { Scielo } \\
\text { /BVS }\end{array}$ & $\begin{array}{l}\text { Modelo APGAR de } \\
\text { Família. }\end{array}$ & $\begin{array}{l}\text { O objetivo do estudo foi analisar as propriedades psicométricas do APGAR de } \\
\text { Família com amostra de } 430 \text { idosos do } \\
\text { Município de Fortaleza-CE, Brasil. }\end{array}$ \\
\hline $\begin{array}{l}\text { Nascimento } \\
\text { LC, et al. }\end{array}$ & 2005 & Qualitativa & $\begin{array}{l}\text { Scielo } \\
\text { /BVS }\end{array}$ & $\begin{array}{c}\text { Modelo Genograma } \\
\text { e Ecomapa }\end{array}$ & $\begin{array}{c}\text { Descrever a experiência da utilização desses } \\
\text { instrumentos no levantamento de dados de uma pesquisa qualitativa, } \\
\text { desenvolvida com nove famílias } \\
\text { de crianças com câncer, que utilizou o interacionismo simbólico e a teoria } \\
\text { fundamentada em dados } \\
\text { como referencial teórico-metodológico a fim de compreender as vivências dessas } \\
\text { famílias. }\end{array}$ \\
\hline $\begin{array}{l}\text { Leão LCA, } \\
\text { et al. }\end{array}$ & 2014 & Qualitativa & Scielo & $\begin{array}{l}\text { Entrevista Familiar } \\
\text { Estruturada- EFE. }\end{array}$ & $\begin{array}{l}\text { Investigar a dinâmica de uma família, utilizando o instrumento clínico de avaliação } \\
\text { Entrevista Familiar Estruturada (EFE) para levantar seus aspectos dinâmicos. }\end{array}$ \\
\hline
\end{tabular}

REAS/EJCH | Vol.12(4) | e2614 | DOI: https://doi.org/10.25248/reas.e2614.2020 Página 7 de 15 


\section{Revista Eletrônica Acervo Saúde / Electronic Journal Collection Health ｜ ISSN 2178-2091}

\begin{tabular}{|c|c|c|c|c|c|}
\hline $\begin{array}{l}\text { Souza J, et } \\
\text { al. }\end{array}$ & 2011 & Quantitativa & Scielo & $\begin{array}{l}\text { Famíly Assessment } \\
\text { Device (FAD) } \\
\text { Family Adaptability } \\
\text { and Cohesion } \\
\text { Evaluation Scales } \\
\text { (FACES III) } \\
\text { Family Environment } \\
\text { Scale (FES) } \\
\text { Family Assessment } \\
\text { Measure (FAM) }\end{array}$ & $\begin{array}{l}\text { Verificar quais instrumentos tem sido utilizado na avaliação do funcionamento } \\
\text { familiar no âmbito da saúde mental. }\end{array}$ \\
\hline $\begin{array}{l}\text { Barbosa } \\
\text { MAM, et al. }\end{array}$ & 2012 & Qualitativa & Scielo & $\begin{array}{l}\text { Modelo de Cuidado } \\
\text { Centrado } \mathrm{Na} \\
\text { Família - CCF }\end{array}$ & Reflexão teórica e filosófica do cuidado centrado na família. \\
\hline $\begin{array}{l}\text { Tallon MM, } \\
\text { et al. }\end{array}$ & 2015 & Qualitativa & PubMed & $\begin{array}{l}\text { Modelo de Cuidado } \\
\text { Centrado } \mathrm{Na} \\
\text { Família - CCF }\end{array}$ & $\begin{array}{c}\text { Discutir teorias e resultados de pesquisas de uma ampla gama de disciplinas, } \\
\text { incluindo epidemiologia, psicologia, sociologia, antropologia e neurociência, foram } \\
\text { acessados para esta discussão }\end{array}$ \\
\hline $\begin{array}{l}\text { Kanervisto } \\
\text { M, et al. }\end{array}$ & 2007 & Quantitativa & PubMed & $\begin{array}{l}\text { Questionário da } \\
\text { Dinâmica Familiar }\end{array}$ & $\begin{array}{c}\text { Descrever a dinâmica familiar em famílias com doença pulmonar obstrutiva } \\
\text { crônica (DPOC) grave, com base na estrutura de Barnhill para sistemas familiares } \\
\text { saudáveis. }\end{array}$ \\
\hline $\begin{array}{l}\text { Johanssoni } \\
\qquad \text { I, et al. }\end{array}$ & 2006 & Qualitativa & PubMed & $\begin{array}{l}\text { Modelo Teórico de } \\
\text { Abordagens de } \\
\text { enfrentamento dos } \\
\quad \text { familiares }\end{array}$ & $\begin{array}{c}\text { Relatar o desenvolvimento de um modelo teórico de abordagens de } \\
\text { enfrentamento de familiares durante a permanência na unidade de terapia } \\
\text { intensiva do paciente e a subsequente recuperação em casa, realizando uma } \\
\text { análise dos conceitos gerados a partir de dois estudos teóricos empiricamente } \\
\text { fundamentados nessa área. }\end{array}$ \\
\hline $\begin{array}{l}\text { Stepans } \\
\text { MBF e } \\
\text { Knight JR. }\end{array}$ & 2002 & Qualitativa & PubMed & Modelo Neuman & $\begin{array}{c}\text { Demonstrar como o modelo de sistemas de Neuman pode ser aplicado à prática } \\
\text { e à pesquisa de enfermagem através do exame do problema da exposição infantil } \\
\text { à fumaça ambiental do tabaco. }\end{array}$ \\
\hline
\end{tabular}

Fonte: Sousa TV, et al., 2019. Brasil, 2019.

REAS/EJCH | Vol.12(4) | e2614 | DOI: https://doi.org/10.25248/reas.e2614.2020 Página 8 de 15 
Como demonstrado no Quadro 2 existe uma variedade de modelos que avaliam desde um aspecto como a comunicação, até aqueles que analisam toda a estrutura familiar, além disso, foram encontrados modelos mais disseminados e conhecidos, mais isolados, com adaptações e propostas de novos modelos de avaliação familiar.

A seguir apresenta-se os mais diversos modelos de avaliação utilizados por enfermeiros encontrados na literatura, bem como um quadro resumo com características essenciais acerca dos mesmos, no intuito de promover uma relação entre tais instrumentos, buscando semelhanças e diferenças bem como estabelecendo uma relação entre os mesmos.

A avaliação do Modelo Calgary se compõe em três pilares: estrutura, funcionalidade e desenvolvimento familiar, apresentando subcategorias com vistas a avaliar a singularidade de cada família em um tempo determinado (DUHAMEL F e TALBOT LR, 2004; SILVA L, et al., 2009; RADOVANOVIC CAT, et al., 2013) . Portanto, avalia estrutura interna (composição familiar, gênero, orientação sexual, ordem de nascimento, subsistemas e limites), externa (famílias extensas sistemas mais amplos) e o contexto (etnia, raça, classe social, religião, espiritualidade e ambiente).

Os Modelos sustentados na Teoria de Adaptação de Roy avaliam a influência do ambiente familiar, das respostas psicológicas, autogestão, enfrentamento situacional, funcionamento familiar, eficácia terapêutica para controle metabólico e qualidade de vida. Esta teoria foi utilizada para a adaptação de estudos que trazem modelos para a população infantil e sua família cujos quais necessitam se adequar ao esquema terapêutico ou uma rotina de hospitalização (TREMARIN RA, et al., 2009; WHITTEMORE R, et al., 2010).

Este modelo constitui-se de seis etapas: coletar dados sobre o comportamento da família e da criança e sua adaptação; avaliar os estímulos internos e externos que influência a adaptação; diagnóstico situacional; estabelecimento de objetivos; intervenção de acordo com os objetivos traçados; e avaliação da intervenção e o alcance dos objetivos (TREMARIN RA, et al., 2009; WHITTEMORE R, et al., 2010).

O Modelo de saúde da família (FSM) permite uma visão completa e holística da saúde da família, possuindo quatro domínios: manutenção da integridade da família e cuidados da saúde; lidar com o estresse relacionado à saúde; Interações entre o provedor da família e de cuidados com agente estressor; e transições e mudanças de saúde e doença (TOMLINSON PS, et al., 2012).

No Modelo de análise de depressão em cuidadores, por sua vez, fundiu-se uma entrevista semiestruturado incluindo informações básicas do paciente, utilizando uma pontuação a partir da escala Mini-mental para analisar o grau de dependência do mesmo; bem como coletam-se informações sobre o cuidador como grau de proximidade e quantidade de horas semanais destinadas ao cuidado deste paciente (LICONA JFM, et al., 2014).

O Modelo de Resiliência baseia-se na Resiliência do Estresse Familiar, Ajuste e Adaptação, sendo utilizado principalmente em famílias com crianças e idosos, sua avaliação concentra-se no agente estressor que afeta a família e a adaptação da mesma em resposta. A utilização deste modelo consegue identificar recursos e habilidades que podem auxiliar no enfrentamento de problemas, verificar a qualidade do relacionamento entre cuidador e idosos, o suporte familiar e os cuidados diários (YEH P-M e BULL M, 2012).

O Modelo Parental foi construído como um instrumento direcionado a pais, com o objetivo de identificar padrões que moldam os mesmos na interação familiar. Assim, inicialmente verifica-se o que é preciso avaliar, e, posteriormente, aplica-se um questionário que proporciona a coleta de dados.

Ele está ancorado três pilares: Padrões de criação (Pais Rígidos, negociador, indulgente e eventual) com seis questões, Título de afeto (Próximo/caloroso, estreito/próximo, elemental/pouco afeto) com três questões e Diretrizes para o sucesso (promotor - pais que acompanham e auxiliam nas decisões, orientador - colabora e apoia, condescendente - aceita as decisões e atribuição indiferenciada) também com três questões (LICONA JFM, et al., 2014).

O Family Background Questionnarie (FBQ) - Adaptado compõem-se de três questionários: "Questionários dos cuidados paternos", "Questionário dos cuidados maternos" e "Questionário do ambiente familiar", sendo constituído por 179 itens e 22 subescalas, o adaptado constitui-se de 12 itens distribuídos pelos três 
questionários (37 itens sobre os cuidados paternos, 37 sobre os maternos e 50 do ambiente familiar). É aplicado na população de adultos-jovens a fim de obter informações para desenvolver um estudo que relacione as experiências familiares no desenvolvimento da patologia depressiva na fase adulta (MONTEIRO IS e MAIA AC, 2010; CICOLINI G, et al., 2012).

Relativo ao Critical Care Family Needs Inventory - CCFNI existem duas versões deste instrumento, uma forma ampliada com 45 itens e uma versão curta com 12 itens, sendo essa última chamada também de Met Needs Inventory cuja qual foi adaptado. A versão curta e adaptada do CCFNI aborda cinco fatores: a necessidade de apoio, conforto, informação, proximidade e segurança. Foi validada para necessidades de questionar os familiares de pacientes internados em Unidades de Terapia Intensiva (MAETíNEZ SG, et al., 2001).

O Modelo APGAR de Família possibilita uma avaliação em cinco domínios Adaptation (adaptação), Partnership (companheirismo), Growth (desenvolvimento), Affection (afetividade) e Resolve (capacidade resolutiva) - APGAR. O instrumento avalia a funcionalidade familiar independente do ciclo de vida dos membros, porém tem sido mais utilizado para avaliar famílias com crianças-adolescentes e de idosos.

Além disso, tem sido aplicado na ESF por permitir a detecção das disfunções do sistema familiar, proporcionando a fundamentação de intervenções direcionadas ou restabelecimento do equilíbrio das relações existentes no sistema (SILVA MJ, et al., 2014).

O Genograma e Ecomapa têm sido utilizados com muita frequência pela enfermagem, principalmente em associação com o Modelo Calgary, para avaliar o arranjo familiar, dinâmicas, relações dos familiares com a comunidade, laços de consanguinidade, afinidade, e relacionamento sem parentesco (NASCIMENTO LC, et al., 2005).

O genograma é vantajoso no sentido de permitir a análise de barreiras nos padrões de comunicação, proporcionando a identificação de hábitos individuais, possibilita a discussão e exposição de opções de mudanças na família além de prevenir o isolamento de um membro. O ecomapa é um diagrama das relações familiares e da comunidade, dos contatos da família, instituições, serviços de saúde e comunidade religiosa que conduzem à identificação de apoios e suportes disponíveis e sua utilização pela família (NASCIMENTO LC, et al., 2005).

A Entrevista Familiar Estruturada é comumente utilizada pela psicologia, constituindo uma ferramenta que avalia a funcionalidade familiar e conjugal. Avalia 10 dimensões: comunicação, papeis, liderança, manifestação da agressividade, afeição física, interação conjugal, individualização, integração, autoestima e promoção da saúde emocional.

Esta ferramenta deve ser aplicada de forma conjunta, ou seja, com todos os membros da família, buscando analisar a dinâmica familiar para um tratamento psicoterápico com enfoque nas disfunções (LEÃO LCA, et al., 2014). O Family Assessment Device (FAD) tem como teoria de base o Modelo McMaster do funcionamento familiar. Avalia os domínios resolutividade de problema, comunicação, papeis, a responsividade e envolvimentos afetivos, controle do comportamento e funcionamento geral.

Através desta avaliação, somada à descrição de estrutura, organização, padrão de transação entre os membros, permite-se a diferenciação entre família saudável e patológica. O instrumento e aplicável a toda família, para membros maiores de 12 anos, sendo composto por 60 itens, tendo enfoque na saúde mental (SOUZA J, et al., 2011).

O Family Adaptability and Cohesion Evaluation Scales (FACES) é baseado no modelo Circumplexo Sistêmico Familiar e Marital, avaliando a coesão, comunicação e adaptação da família e diferenciando o funcionamento real e ideal. É mais comumente utilizado para avaliar famílias com membros adolescentes e para casais sem filhos (SOUZA J, et al., 2011).

O Family Environment Scale (FES) é um modelo baseado nas teorias socioecológica e psicológica e teoria sistêmica familiar, possibilitando avaliar características do ambiente social de qualquer tipo de família e mudanças advindas da dependência química de algum membro. Contém 90 itens, 10 subescalas, com três dimensões: relacionamento, coesão, expressividade, conflito; crescimento pessoal, independência, 
assertividade, interesses intelectuais, lazer e religião; manutenção do sistema, organização e controle. É aplicável a toda família com membros maiores de 11 anos (SOUZA J, et al., 2011).

O Family Assessment Measure (FAM) enfoca a dinâmica e interação familiar e é usado para diagnosticar resultados da terapia dos processos familiares. Os Modelos de McMaster de Funcionamento Familiar e de Categorias da família são seus referenciais. Desta forma o mesmo enfoca a dinâmica e interação familiar e é usado para diagnosticar resultados da terapia dos processos familiares.

Possui 134 itens em três escalas sendo a geral, a de relacionamento didático e escala de funcionamento individual, e avalia sete dimensões: comprimento de tarefas, desempenho de papeis, comunicação, expressão e envolvimento afetivos, controle, valores e normas. Aplicável a toda a família (SOUZA J, et al., 2011).

O Cuidado Centrado na Família (CCF) é um modelo destinado a utilização em famílias com crianças predominantemente em situação de hospitalização. É composto por oito elementos estruturais: reconhecer a presença constante da família na vida da criança; promover a colaboração nos cuidados à saúde do paciente entre pais e profissionais de saúde; admitir compartilhamento de informações sobre os cuidados de saúde do paciente de forma imparcial, completa e contínua com os pais; prover a instauração de políticas e programas que deem suporte e apoio emocional e financeiro para prováveis necessidades da família.

Identificar, considerando a individualidade e a forma de cuidar, as forças dessa família; entender e considerar as necessidades de desenvolvimento do indivíduo e da família nos vários sistemas de dispensação de cuidado em saúde; estimular e propiciar o apoio entre famílias; garantir a acessibilidade e flexibilidade nos cuidados em saúde, levando em consideração as necessidades individuais de cada família (BARBOSA MAM, et al., 2012; TALLON MM, et al., 2015).

O Questionário de Dinâmica Familiar foi utilizado para coleta de dados sobre a dinâmica familiar. Foi traduzido do Inglês para o Finlandês e depois foi novamente traduzido parao Inglês. É um questionário com 66 itens, que fora utilizado para medir dados sociodemográficos como: idade, sexo, estrutura familiar, educação e doenças com influência na família. Tem como referencial teórico o autor Barnhill (KANERVISTO M,et al., 2007).

O Modelo Teórico de Abordagens de Enfrentamento dos Familiares enfoca a avaliação de familiares com pacientes internados na UTI. É um modelo não validado pouco estruturado e que primariamente e de uma forma geral avalia o enfrentamento situacional da família em relação ao seu familiar internado na UTI (JOHANSSONI I, et al., 2006).

O Modelo de Sistemas de Neuman defende que fatores estressores intrapessoais, interpessoais e extrapessoais irão ditar o nível de adaptação do indivíduo as diversas situações. Avaliam famílias com crianças, adolescentes e idosas, sobretudo em situação de hospitalização e agravo à saúde.

Estrutura-se em determinadas características e fatores que influenciam as mesmas, e a partir da coleta de dados possibilita o desenho de uma intervenção personalizada, aplicável àquela, possivelmente mais eficaz, considerando as diversas realidades, situações e conjunturas familiares (STEPANS MBF, et al., 2002; CARVALHO FILHA FSS, et al., 2019).

Assim, como mencionado, baseando-se em informações importantes acerca de todos estes Modelos de Avaliação, implementou-se um resumo das principais características, com vistas a se discutir sobre cada instrumento, destacando a sua importância e uso para a Enfermagem (Quadro 3). 
Quadro 3 - Características individuais dos Modelos Teóricos utilizados pela Enfermagem para avaliação das famílias. Brasília-DF, Brasil, 2019.

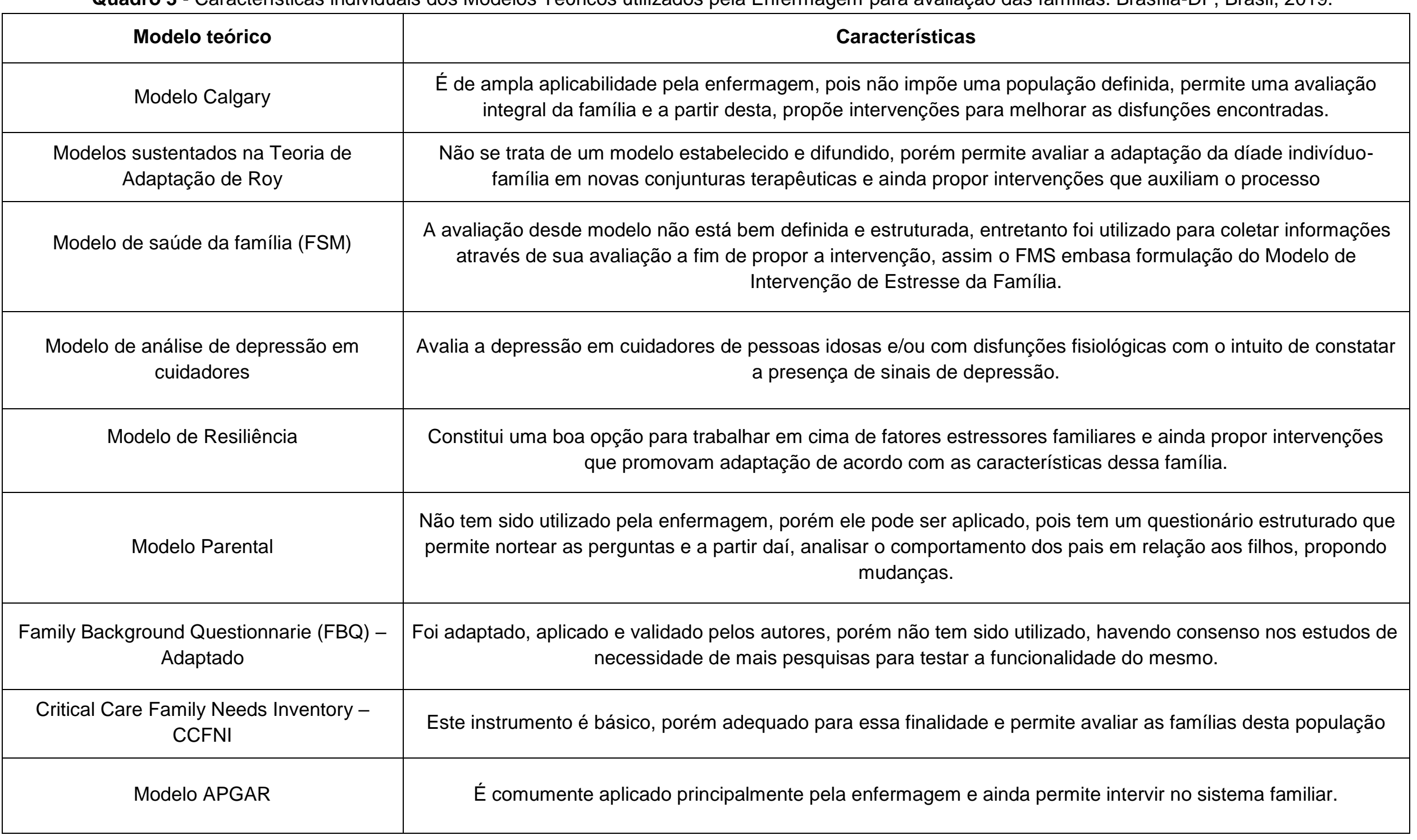

REAS/EJCH | Vol.12(4) | e2614 | DOI: https://doi.org/10.25248/reas.e2614.2020 Página 12 de 15 


\begin{tabular}{|c|c|}
\hline Genograma e Ecomapa & $\begin{array}{l}\text { São frequentemente utilizados pela enfermagem, permitindo uma visibilidade ampla das relações, além de } \\
\text { estudar a busca de suporte e possibilita a criação de vínculo entre o profissional e o familiar. }\end{array}$ \\
\hline Entrevista Familiar Estruturada & $\begin{array}{l}\text { É pouco utilizado pela enfermagem, porém serve de referencial para realizar uma avaliação familiar ou até } \\
\text { mesmo estruturar um modelo. }\end{array}$ \\
\hline Famíly Assessment Device (FAD) & $\begin{array}{l}\text { Pode ser usado pela enfermagem desde que seja em uma avaliação da família mais focalizada para saúde } \\
\text { mental. }\end{array}$ \\
\hline $\begin{array}{l}\text { Family Adaptability and Cohesion } \\
\text { Evaluation Scales (FACES) }\end{array}$ & $\begin{array}{l}\text { É mais conhecido pelo uso em saúde mental, avaliando a funcionalidade familiar. Pode ser utilizado por toda } \\
\text { enfermagem, principalmente na Atenção Básica em qualquer tipo de família. }\end{array}$ \\
\hline Family Environment Scale (FES) & $\begin{array}{l}\text { É pouco utilizado pela enfermagem, mas pode ser aplicada em familiares com dependentes químicos no intuito } \\
\text { de analisar a mudança desse aspecto. }\end{array}$ \\
\hline Family Assessment Measure (FAM) & $\begin{array}{l}\text { Dentre os modelos utilizados pela saúde mental, este é o menos utilizado. Avalia sete dimensões, como o } \\
\text { modelo de FAD, porém é mais extenso demandando mais tempo durante a coleta de dados. Pode ser utilizado } \\
\text { pela enfermagem e em qualquer tipo de família. }\end{array}$ \\
\hline Cuidado Centrado na Família (CCF) & $\begin{array}{l}\text { É um modelo que estimula o potencial e promove o empoderamento familiar, auxiliando à família com paciente } \\
\text { hospitalizado na tomada decisão acerca das intervenções. }\end{array}$ \\
\hline Questionário de Dinâmica Familiar & $\begin{array}{l}\text { Não foi encontrado evidências sobre a utilização desse modelo aqui no Brasil. Ele apresenta um questionário, } \\
\text { que pode ser adaptado e utilizado pela enfermagem. }\end{array}$ \\
\hline $\begin{array}{l}\text { O Modelo Teórico de Abordagens de } \\
\text { Enfrentamento dos Familiares }\end{array}$ & $\begin{array}{l}\text { Não apresenta nenhuma evidência de ser utilizado pela enfermagem, mas acredita-se ser possível fazer estudos } \\
\text { e adaptação para o cenário do Brasileiro, pela importância e a necessidade se avaliar o enfrentamento } \\
\text { situacional da família com um ente internado na UTI. }\end{array}$ \\
\hline Modelo de Sistemas de Neuman & $\begin{array}{l}\text { Aplicado à família, pretende investigar o que e quais são os fatores estressores para essa família a fim de ditar } \\
\text { melhores intervenções no sentido de aumentar o padrão de adaptabilidade as situações de agravo à saúde. }\end{array}$ \\
\hline
\end{tabular}

Fonte: Sousa TV, et al., 2019.

REAS/EJCH | Vol.12(4) | e2614 | DOI: https://doi.org/10.25248/reas.e2614.2020 Página 13 de 15 
Dos estudos, 70\% (14) da amostra se baseiam em estudos qualitativos, ademais falta a operacionalização de estudos quantitativos ou estudos mistos para padronização e aferição de fenômenos que acometem as famílias na ESF pelos enfermeiros. Desta forma os modelos se mostram complexos, extensos e de difícil aplicação na realidade estudada.

\section{CONSIDERAÇÕES FINAIS}

São mais comumente utilizados na prática clínica do enfermeiro no atendimento às famílias na ESF o Modelo Calgary, o APGAR de família e o Genograma e Ecomapa. Durante sua assistência, os enfermeiros precisam identificar as situações que ameaçam as famílias utilizando de modelos teóricos de avaliação de família para propiciar a melhoria na qualidade do cuidado dispensado, bem como a sistematização da assistência, a integração da família e suas relações no cuidar. Para além disso, compreende-se a urgência de enfermeiros conhecerem e apropriarem-se de modelos teóricos de avaliação familiar, principalmente na Atenção Básica, no intuito de individualizar a atenção, qualificar o cuidado, fortalecer vínculos e promover novas e instigantes formas de cuidar e ser cuidado.

\section{REFERÊNCIAS}

1. BARBOSA MAM, et al. Cuidado centrado na família no contexto da criança com deficiências e sua família: uma análise reflexiva. Text Context Enferm, 2012;21(1):194-199.

2. CARVALHO FILHA FSS, et al. Uso de instrumentos para triagem e diagnóstico dos transtornos do espectro do autismo: revisão integrativa. Intern J Development Research, 2019;09(10):30356-30362.

3. CHRISTOFFEL MM, et al. Modelo Calgary de avaliação da família de recém-nascidos: estratégia pedagógica para alunos de enfermagem. Esc Anna Nery Rev Enferm, 2008;12(1):160-165.

4. CICOLINI G, et al. Influence of family carers on haemodialyzed patients' adherence to dietary and fluid restrictions: an observational study. J Advanc Nurs, 2012;68(11):2410-2417.

5. COVINSKY KE, et al. Patient and Caregiver Characteristics Associated with Depression in Caregivers of Patients whith Dementia. J Gen Intern Med, 2003;18(0):1006-1014.

6. DUHAMEL F,TALBOT LR. A constructivist evaluation of family systems nursing interventions with families experiencing cardiovascular and cerebrovascular. J Family Nurs, 2004;10(1):12-32.

7. FIGUEIREDO MHJS, MARTINS MMFS. Avaliação Familiar do Modelo Calgary de Avaliação da Família aos Focosda Prática de Enfermagem. Cienc Cuid Saúde, 2010;9(3):552-559.

8. FILHO IM de M, et al. Avaliação do estresse ocupacional de enfermeiros da Estratégia Saúde da Família. Rev. G\&S. $12^{\circ}$ de agosto de 2019;9(3):335-43.

9. FILIZOLA CLA, et al. A história da família de Rubi e seu filho Leão: trabalhando com famílias de usuários com transtorno mental grave através do Modelo Calgary de avaliação e intervenção na família. Text Context Enferm, 2003;12(2):182-190.

10. JOHANSSONI I, et al. Theoretical model of coping among relatives of patients in intensive care units: a simultaneous concept analysis. J Advan Nurs, 2006; 56(5):463-471.

11. KANERVISTO M, et al. Family dynamics in familes of severe COPD patients. J Clin Nurs, 2007;16:1498-1505.

12. LEÃO LCA, et al. Avaliação Clínica de relações familiares com a utilização da Entrevista Familiar Estruturada (EFE): Estudo de Caso. Psicologia da Saúde. 2014;22(1):1-7.

13. LICONA JFM, et al. Study of parentalmodels: building na instrumento for their exploration. Rev Esc Enferm USP, 2014; 48(4):593-600.

14. LOPES MSL, MARCON SS. A hipertensão arterial e a família: necessidade do cuidado familiar. Rev Esc Enferm USP, 2009;43(2):343-350.

15. MAETÍNEZ SG,et al. El Cuestionario de Necesidades de los Familiares de Pacientes de Cuidados Intensivos (CCFNI) version breve: adaptación y validación em población española. An Sist Sanit Navar, 2001;34(3):349-361.

16. MENDES KDS, et al. Revisão Integrativa: método de pesquisa para a incorporação de evidência na saúde e na enfermagem. Texto contexto, Florianópolis, 2008 Out-Dez;17(4):758-64.

17. MONTEIRO IS, MAIA AC. Avaliação psicométrica de três questionários sobreo historial familiar. Rev Psiq Clín, 2010;37(3):97-104.

18. FILHO IM de M, et al. Avaliação do estresse ocupacional de enfermeiros da estratégia saúde da família. Rev. G\&S. 2018;9(3):335 -43.

19. MORAES IMF, et al. Associação de estresse ocupacional e uso de psicotrópicos por docentes da área da saúde. Rev Bras Promoç Saúde. 2019;32:9007.

20. NASCIMENTO LC, et al. Contribuições do Genograma e do Ecomapa para o Estudo De Famílias em Enfermagem Pediátrica. Texto Contexto Enferm, 2005;14(2):280-286.

21. RADOVANOVIC CAT, et al. Avaliação Estrutural, Desenvolvimental e Funcional da Família de Indivíduos com Hipertensão Arterial. Rev Gaúcha Enferm, 2013:34(1):45-54. 
22. SEOANE AF, FORTES PAC. A percepção do usuário do programa saúde da família sobre a privacidade e a confidencialidade de suas informações. Saúde Soc. 2009;18(1):42-9.

23. SILVA L, et al. Aplicação do Modelo Calgary para avaliação de famílias de idosos na prática clínica. Rev Bras Enferm, 2009; 62(4):540-534.

24. SILVA MJ, et al. Análise psicométrica do APGAR de família. Esc Anna Nery, 2014;18(2): 527-532.

25. SOUZA J, et al. Avaliação do Funcionamento familiar no contexto da Saúde Mental. Rev Psiq Clín, 2011; 38(6): 254259.

26. STEPANS MBF, KNIGHT JR. Application of Neuman's framework: infant exposure to environmental tabacco smoke. Nurs Scien Quarterly, 2002; 14(4):327-334.

27. TALLON MM, et al. Rethinking Family-centred care forthe child and Family in hospital. J Clinic Nurs, 2015; 24:14261435.

28. TOMLINSON PS, et al. A family systems nursing intervention model for paediatric health crisis. J of Advanced Nursing, 2012;68(3):705-714.

29. TREMARIN RA, et al. A Teoria da Adaptação Sustentando o Cuidado de Enfermagem em Hospital Pediátrico: Um Estudo de Caso. Cogitare Enferm, 2009;14(3):569-574.

30. WHITTEMORE R, et al. A Conceptual Model of Childhood Adaptation to Type 1 Diabetes. Nurs Outlook, 2010;58(5):242-251.

31. WRIGHT LM, LEAHEY M. Avaliação e Intervenção na Família. In: Wright LM, Leahey M. Enfermeiras e Famílias. Um guia para avaliação e intervenção na família. São Paulo: Roca; 2012b.

32. YEH PM, BULL M. Use of the resiliency modelo $f$ family stress, adjustment and adaptation in the analysis of family caregive reaction among families ofolder people whith congestive heart failure. International Jornal of Older People Nursing, 2012;7:117-126. 